\title{
A New Technique of Sentinel Lymp Nodes Detection in Vulvar Cancer Patients. The SARVU Study.
}

\section{Jedryka M, Kryszpin M, Manowiec M, Hirowska-Tracz M, Rossochacka B \& Kalus M, Klimczak P \\ Lower Silesian Oncology Center, Wroclaw, Poland \& Opole Oncology Center, Opole, Poland}

\section{Objectives}

We have created the SARVU study (Sentinel Lymph Nodes Detection with Sentimag against Radiotracer in Vulvar Cancer) to compare and validate the use of ferromagnetic technique of SLN detection with iron oxide tracer (Sienna+ß) and magnenometer probe (Sentimag®) Vs. standard procedure with radioisotope (99mTc and gamma probe in vulvar cancer patients.

\section{Materials and Methods}

We included 20 patients with squamous vulvar tumour less then $4 \mathrm{~cm}$ and negative lymph nodes in imaging pre-op work-up. The primary endpoint was the propotion of succesful SLN detection with Siena+® vs. 99mTc. The secondary endopoints were: the average of SLN per patient, the proportion of SLN detected (nodal detection rate), the propotion of pathologically positive results (malignancy rate) per patient and per node
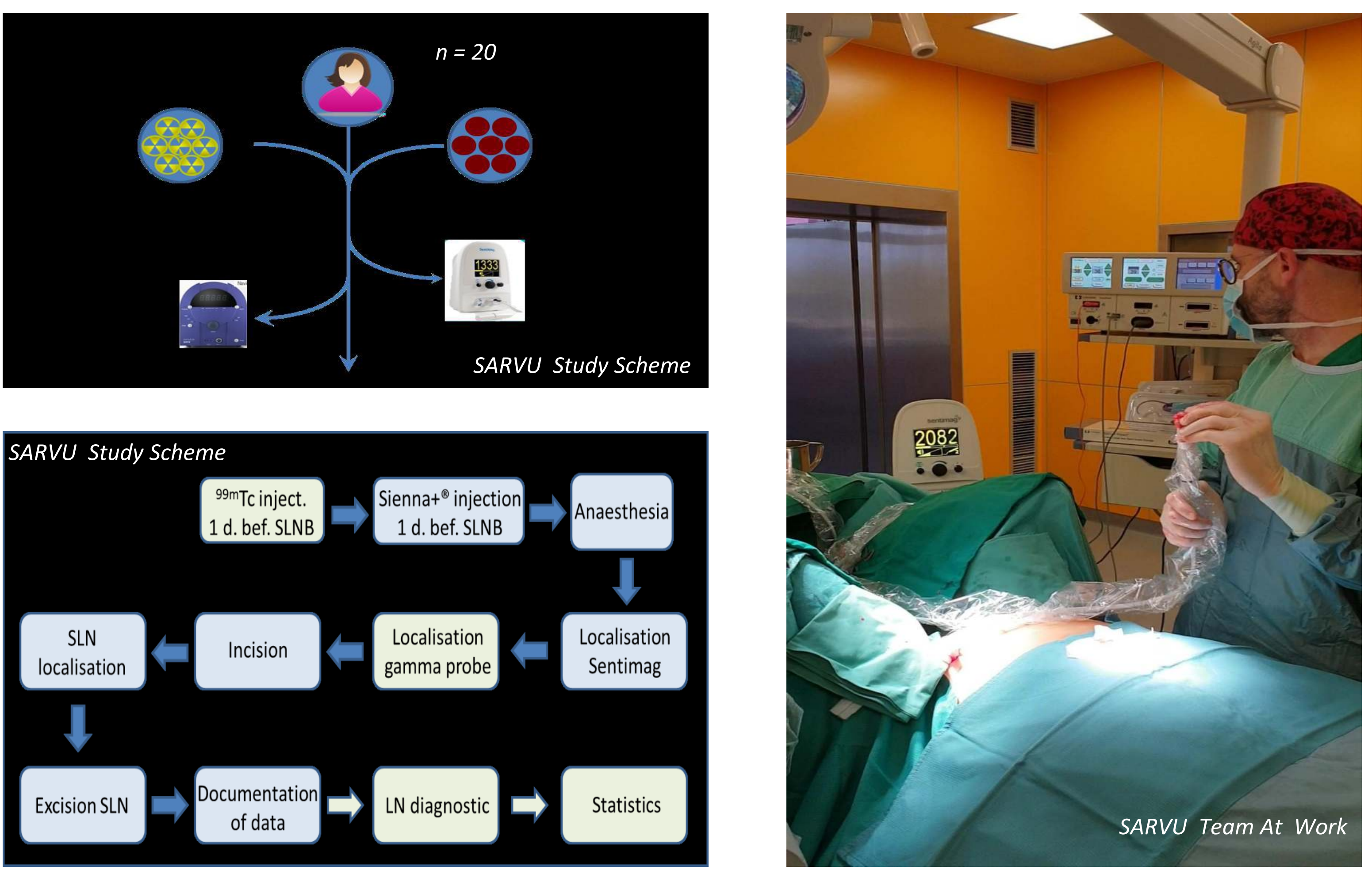

\section{Results}

\begin{tabular}{|c|c|c|}
\hline & Radiotracer ${ }^{\left({ }^{99} \mathbf{T c}\right)}$ & Ferromagnetic (Sienna+ ${ }^{\boldsymbol{}}$ ) \\
\hline Number of patients with SLN detected & 20 & 64 \\
\hline Number of SLN detected -overall & 61 & 3,2 \\
\hline Number of SLN/per patient & 3,1 & 1 \\
\hline Number of SLN missed & 4 & $100 \%$ \\
\hline SLN detection rate/per patient & $100 \%$ & $98,5 \%$ \\
\hline SLN detection rate/per node & $93,8 \%$ & 14 \\
\hline Number of metastatic SLN & 14 & 9 \\
\hline Number of patients with metastatic SLN & 9 & $21,5 \%$ \\
\hline Malignancy rate per node & $21,5 \%$ & $45,0 \%$ \\
\hline Malignancy rate per patient & $45,0 \%$ & $100 \%$ \\
\hline Maligancy detection rate/per node & $100 \%$ & $100 \%$ \\
\hline Malignancy detection rate/ per patient & $100 \%$ & \\
\hline
\end{tabular}

\section{Conclusions}

We consider the new method of SLN detection with the use of ferromagnetic injection in vulvar cancer patients as reliable, safe and non-inferior to the standard of care with a radiocoloid 